\title{
Perceptual grouping in visual word recognition
}

\author{
J. M. BOCK, A. F. MONK, and C. HULME \\ University of York, York, England
}

\begin{abstract}
Four experiments are presented in which printed texts are read for their meaning. Some of the texts were mutilated by altering the size of selected letters. In Experiments 1, 2, and 3, the number of words mutilated per passage and the number of letters changed per word were both manipulated. In all three experiments, reading was slowed as a function of the number of words changed per passage, while the number of letters changed per word had a much smaller effect. The interaction between the number of words and number of letters changed was not significant in any of the experiments. It is difficult to explain these results merely in terms of changes in the discriminability of letters. In Experiment 2 all uppercase text was used, which argues against an explanation in terms of supraletter features such as word envelope. We propose an explanation in terms of visual attention and the perceptual grouping required prior to feature recognition. The last experiment supports this explanation through the counterintuitive finding that adding letters of intermediate size can improve legibility by allowing grouping processes to associate large and small letters as belonging to the same word object.
\end{abstract}

In the experiments presented in this paper, we were concerned with how visual information is used to mediate word recognition during the fluent reading of text. Most studies of the processes involved in word recognition in reading have involved experimental tasks in which readers are presented with isolated words (see, e.g., Taft, 1991, for a recent review of many such studies). Ultimately, however, the researcher's goal in this area must be to understand the processes involved in the reading and understanding of text, and there are good theoretical reasons to suppose that the types of visual information that are important in the reading of text differ from those involved in the recognition of individual words. Specifically, in the latter case, the reader's attention is controlled by the experimenter. A single word stimulus is presented in a predictable, foveally central, position. This removes from the reader the necessity of guiding visual attention, which is an important part of the task of reading continuous texts.

One area of research in which fluent reading is the basic experimental task is the work done with eye-movement contingent displays (see, e.g., Rayner \& Pollatsek, 1989). The extensive work of McConkie and his colleagues led McConkie and Zola (1987) to propose a four-level object hierarchy that guides visual attention when one is reading a continuous text. Early in eye fixation, the visual system provides a representation of the full page of text, organized as a spatially indexed object hierarchy. This is a rapid, parallel process. The information gathered is not used for either lexical or semantic access. Rather, it is a spatial guidance system. McConkie and Zola propose that attention may be given to objects at various levels

Correspondence should be addressed to A. Monk, Department of Psychology, University of York, York YO1 SDD, England. within the hierarchy. On initial fixation of the page, orientation might require attention at the page level. During the initial, or final, fixations of a line, line-level objects may be attended to as the reader orients to the following line. Typically, the reader attends to whole-word patterns as objects. During this stage, information is used for lexical access. However, if a word is not readily identified, attention to objects at the letter level may be necessary.

McConkie and Zola's (1987) analysis raises a number of interesting questions. In particular, before attention can be directed to some level in their object hierarchy, the visual stimulus must be appropriately segmented into lines on the page, words in the line, and, when necessary, letters in the word. Reading theorists have generally ignored this problem. However, segmentation and grouping have been studied in the context of other visual tasks.

The perceptual grouping of parts into wholes was covered most notably by the Gestalt psychologists (Koffka, 1935; Kohler, 1947; Wertheimer, 1923). They formulated laws of perceptual organization, rules that described which attributes of the visual pattern would cause either grouping of parts into wholes or segregation of the visual array. Two examples of these laws are the law of proximity and the law of similarity. The law of proximity states that items that are relatively close together in the visual field will be grouped together. The law of similarity states that items that share similar attributes (e.g., color, brightness, or form) are more likely to be grouped together than are those that do not.

More recent research in this area has emphasized the relationship between perceptual grouping and the focus of attention. Grouping is seen to be an automatic process whose function is to mediate attention (Kahneman \& Henik, 1975; Treisman, 1982). Thus, Pomerantz (1981) defined perceptual grouping operationally, as a "failure of 
selective attention" to the parts that compose the group. The argument is that because grouping forces attention to the whole, it is concomitantly harder to attend selectively to the parts that constitute that whole.

In reading, grouping might be expected to be achieved through the proximity and similarity of letters. In normal texts, interletter spacing is smaller within words than between words, allowing grouping by proximity. There are a number of ways in which letters in the same font are similar. The most obvious is similarity of scale, which has been suggested as a major determinant of grouping in other visual tasks (Marr, 1980). Scale is also known to have important effects on visual attention (Kimchi, 1988; Navon, 1977).

If the scale of letters and their proximity determines grouping, then manipulations that affect these variables might be expected to reduce legibility. Mutilating continuous text by changing the case or size of selected letters reduces its legibility (Rudnicky \& Kolers, 1984; Smith, 1969; Smith, Lott, \& Cronnell, 1969). These effects have been traditionally interpreted in terms of feature recognition. Thus interspersing uppercase letters in a lowercase word will disrupt word shape and may also obscure features such as the relative height of letters. However, because these experiments have been done with continuous text, the observed reductions in legibility could equally well be explained in terms of the disruption of grouping processes and consequent interference with the direction of visual attention. In the present experiments, we explored the effects of changing the size of letters within texts, and we attempted to relate these effects to the role of visual grouping and attentional mechanisms in fluent reading.

\section{EXPERIMENT 1}

Rudnicky and Kolers (1984, Experiment 2) found that reading was slowed if strings of case-alternated text, of various lengths, were inserted within passages printed in lowercase. An a posteriori analysis of the data for individual passages revealed that case alternation decreased reading speed in proportion to the number of words disrupted rather than in proportion to the number of letters changed in case. This result is interesting, because it is difficult to explain on the basis of letter features' simply being obscured in some way. However, it is hard to accept their results at face value, because they depend on an a posteriori analysis of the data. Moreover, Rudnicky and Kolers used text that changed in case rather than just size.

In Experiment 1, we sought to replicate this effect of word-pattern disruption within a planned experiment. The text was printed normally in lowercase; capitals were used only at the beginning of sentences and for proper nouns; and only the size of letters was changed, not their case. Either 125 or 250 words within each text (mean length, 411 words) were disrupted through increases in the size of their constituent letters. A fully crossed design was used, yielding the following conditions: (1) a control condition of lowercase type printed in 9-point typeface;
(2) one letter increased in size in 125 words; (3) one letter increased in size in 250 words; (4) two letters increased in size in 125 words; and (5) two letters increased in size in 250 words.

It was anticipated that all the experimental passages would be read more slowly than would those in the control condition. However, the main point of interest was whether this slowing of reading would be due principally to the number of letters that were increased in size within a word, or to the number of word patterns that were disrupted. To assess this we could compare the reading speeds of texts with the same number of words disrupted, but the texts differed in the number of letters changed in size.

\section{Method}

Subjects. Twenty-five adult skilled readers participated in the experiment. Fifteen were male and 10 were female. Their ages ranged from 21 to 40 years. They were all students from a postgraduate course in information technology and gave their time freely.

Design. The subjects were required to read five passages of text that had been altered according to the experimental conditions. This involved producing 25 texts. Each subject performed all five conditions. The order of presentation was balanced with the use of five $5 \times 5$ Latin squares; the texts within each condition were balanced across the squares, with each text presented once only within each line of the square.

Materials. Texts. The passages of text were taken from the Micropaedia Brittanica. Each had as its subject an infectious illness. They had a mean length of 411 words. Only letters within words of four letters or more were changed in size. Each passage contained 250 to-be-changed words and fitted onto one side of A4 paper.

Typesize and font. Figure 1 presents extracts from the texts used in each of the five experimental conditions. The main body of the text was printed in a font size of 9 points, with the letters that were increased in size printed in 18 points. This ratio of difference, $2: 1$,

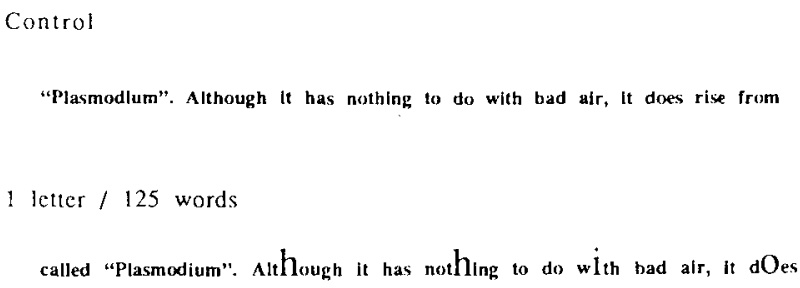

Figure 1. Samples from the materials used in Experiment 1. 
has previously been found to retard reading rate significantly (Rudnicky \& Kolers, 1984, Experiment 3). The font used was Times, with a line spacing of 20 points. The 18-point stimuli were printed in normal roman, and the 9-point stimuli in boldface, to equate the weight of the font sizes. The passages were prepared on an Apple Macintosh with Pagemaker Version 2 (Aldus) and an Apple LaserWriter Plus. Letter spacing was adjusted to a maximum of $1 \mathrm{~mm}$, and the minimum word spacing was $2 \mathrm{~mm}$. There was a spacing variation of $.5 \mathrm{~mm}$; letter spacing varied between .5 and $1 \mathrm{~mm}$, while word spacing varied between 2 and $2.5 \mathrm{~mm}$. So that letter position could not be confounded with size change, all the letters to be changed were distributed randomly within words.

Comprehension sheets. A comprehension test was inserted as the next sheet in the test booklet after each experimental text. The comprehension sheet contained 10 questions, 5 of which could be answered on the basis of the passage that had just been read, and 5 of which could not. Following the methodology of Rudnicky and Kolers (1984), the subject's task was to tick the 5 questions they thought they could answer on the basis of reading the passage. Since there were five passages of text, five appropriate comprehension sheets were designed.

Procedure. Each subject was presented with the five passages of text, in a booklet with a comprehension sheet inserted behind each passage. Each subject was given a stopwatch to measure the time taken to read each passage of text. Silent reading times were gathered, because reading aloud has been found to set a floor on reading rate data (Rudnicky \& Kolers, 1984; Smith et al., 1969).

Before Experiment 1 was performed, the comprehension tests were validated in a small subexperiment. Ten subjects drawn at random from the university campus were asked to study the comprehension tests. The subjects were asked to guess which five questions were the correct ones in relation to the subject matter of the text, given only the title of the text. The results showed that subjects could only guess, at best, one or two of the five correct answers. A criterion for adequate comprehension in the following experiments was set at one question correct above this subexperiment highest score (three questions correct for four passages and two for the other).

In Experiment 1, 2 subjects failed to complete the comprehension questions properly, and 1 scored lower than criterion on Conditions 2 and 4 . The appropriate three lines within the Latin square were reallocated to new subjects, whose scores were substituted.

\section{Results}

Table 1 includes the mean comprehension scores for each condition (out of five). There was no significant difference between these conditions $[F(4,96)=.87$, n.s.]. Any differences in reading speed between conditions, therefore, cannot be attributed to differences in the extent to which subjects read for meaning.

Mean reading rates, expressed in words per minute for each condition, are also given in Table 1 . They are significantly different $[F(4,96)=18.78, p<.0001]$. An a posteriori comparison between the reading times per condition revealed that, as anticipated, the control condition was read significantly more quickly than each of the experimental conditions (HSD $=17.51, p<.05$ ).

The main point of interest here, however, is the degree to which reading rate slows down because of letter-feature disruption in comparison with the degree to which it slows down because of disruptions to whole-word patterns. To explore this, we transformed the data into difference
Table 1

Mean Reading Rate in Words per Minute and Comprehension Scores for Each Condition in Experiment 1

\begin{tabular}{lccccc}
\hline & \multicolumn{2}{c}{ Words/Minute } & & \multicolumn{2}{c}{ Comprehension } \\
\cline { 2 - 3 } \cline { 5 - 6 } Condition & $M$ & $S D$ & & $M$ & $S D$ \\
\hline Control & 260.90 & 82.71 & & 4.24 & .88 \\
1L/125W & 219.52 & 76.22 & & 4.00 & .76 \\
1L/250W & 209.70 & 79.98 & & 4.04 & .74 \\
2L/125W & 217.93 & 74.80 & 3.92 & .70 \\
2L/250W & 197.04 & 74.80 & & 3.92 & .91 \\
\hline
\end{tabular}

Note-1L, one letter changed in size per word. 2L, two letters changed in size per word. $125 \mathrm{~W}, 125$ words changed per text. $250 \mathrm{~W}, 250$ words changed per text. $N=25$.

scores, representing the difference between performance in the control condition and in each experimental condition. These scores were entered into a two-way withinsubject analysis of variance (ANOVA). The first factor was the number of letters changed in size per word; the second factor was the number of words changed per text. A significant main effect was found for the number of words in which letters were changed in size within each text $[F(1,24)=7.17, p<.01]$. However, the main effect for the number of letters changed per word was not found to be significant $[F(1,24)=1.48$, n.s. $]$, nor was there any significant interaction between the number of letters changed in size and the number of words that the size changes affected $[F(1,24)=1.57$, n.s.]. It appears that changing the size of letters within words slows reading rate by disrupting the integrity of whole-word patterns, much more than by disrupting letter-feature information.

\section{Discussion}

Changing the size of letters within words slows reading rate significantly. The more words that are disrupted by changes, the more reading is slowed. In contrast, the number of letters changed within a word has a much smaller effect on reading rate.

This finding contrasts sharply with the predictions of feature-based models of visual word recognition (e.g., McClelland \& Rumelhart, 1981; Rumelhart \& McClelland, 1982). Such models state that reading entails the preliminary recognition of letters within words, and that letter recognition occurs through feature analysis. Increasing the relative size of letters within words should therefore obscure information about the letter features. On this basis, it would be anticipated that reading should slow down as the number of letters that are changed in relative size increases. This slowing might be due to either featural lack of clarity or the time taken to rescale the changed letters to the same size as the main body of the word (Larsen \& Bundesen, 1978; Rudnicky \& Kolers, 1984). One possible interpretation of the finding that reading slows in proportion to the number of words that are disrupted by letter-size changes is that word reading is slowed because of disruption to some supraletter feature such as the whole-word pattern. An alternative explanation is that 
the size changes disrupt grouping processes so that attention cannot be appropriately directed. Experiment 2 is designed to test these alternatives.

\section{EXPERIMENT 2}

It has long been argued that the shape of words might act as an additional cue to recognition, as a supraletter feature (e.g., Cattell, 1886; Monk \& Hulme, 1983; Tinker, 1963). Such a suggestion remains highly contentious, however (Paap, Newsome, \& Noel, 1984). Word shape is peculiar to words printed in lowercase, and it can be defined as the pattern of ascending and descending information inherent to each word. Because word shape is only present in lowercase words, there should be a superiority in processing lowercase words as opposed to uppercase words. This is indeed so; when the reading speeds of lower- and uppercase texts are compared, lowercase is found to be read more quickly (Tinker, 1963).

It is extremely difficult to create experiments in which the variables of letter features, word shape, and wholeword structure can be independently manipulated. For example, in the experiment cited above (Tinker, 1963), lowercase text was read more quickly than uppercase, and this was attributed to the extra cue of word shape. However, a letter-feature theorist could argue that this difference was due to the heightened discriminability of letters in lowercase text, rather than to the overall shape of the word.

Experiment 1 provides us with a way of separating the effect of letter disruption in a realistic reading task by comparing the effects due to the number of letters changed in size per word with the effect of the number of word patterns that have been disrupted within the texts. We can test the word-shape hypothesis by repeating the first experiment with texts that do not have cues to word shapethat is, texts in uppercase. If, using uppercase text, we should find a result opposite that of Experiment 1, and if reading should slow because the number of letters changed in size per word increased, then this would suggest that the unique shape of lowercase words was exerting an influence in the first experiment that was not present in the second. Conversely, if the results of Experiment 1 should be replicated with the use of uppercase text, and if reading should slow to a degree consistent with the number of word patterns disrupted within the text, rather than the number of letters changed per word, then this would present a problem for both feature-based theories and word-shape, or outline, hypotheses. In this case, the whole pattern of a word would be shown to be of importance in reading, but this could have nothing to do with a cue for word shape or outline information.

\section{Method}

The method was the same as that for Experiment 1. The passages of text were the same as those used in the first experiment. They differed only in that they were printed in uppercase rather than lowercase. Twenty-five adult skilled readers participated in the experiment. Fifteen were male, and 10 were female. Their ages ranged from 19 to 35 years. Fifteen were 2nd-year psychology under-
Table 2

Mean Reading Rate in Words per Minute and Comprehension Scores for Each Condition in Experiment 2

\begin{tabular}{|c|c|c|c|c|}
\hline \multirow[b]{2}{*}{ Condition } & \multicolumn{2}{|c|}{ Words/Minute } & \multicolumn{2}{|c|}{ Comprehension } \\
\hline & $M$ & $S D$ & $M$ & $S D$ \\
\hline Control & 233.78 & 66.75 & 4.20 & .65 \\
\hline $1 \mathrm{~L} / 125 \mathrm{~W}$ & 203.99 & 45.66 & 4.12 & .83 \\
\hline $1 \mathrm{~L} / 250 \mathrm{~W}$ & 179.51 & 36.49 & 4.12 & .88 \\
\hline $2 \mathrm{~L} / 125 \mathrm{~W}$ & 195.09 & 46.07 & 3.92 & .76 \\
\hline $2 \mathrm{~L} / 250 \mathrm{~W}$ & 178.65 & 34.41 & 4.40 & .71 \\
\hline
\end{tabular}

Note-1L, one letter changed in size per word. $2 \mathrm{~L}$, two letters changed in size per word. $125 \mathrm{~W}, 125$ words changed per text. $250 \mathrm{~W}, 250$ words changed per text. $N=25$.

graduates who gave their time freely. Ten were electronics undergraduates who were paid $£ l$ each. Two subjects were rejected because their comprehension test scores fell below the criterion of three correct responses per text. Their lines within the Latin square were reallocated to new subjects and rerun.

\section{Results}

Table 2 includes the mean comprehension scores per condition. There are no significant differences between these means $[F(4,96)=1.33$, n.s.]. Mean reading rates for this experiment, expressed in words per minute, are also given in Table 2. As one would expect, these are slower than in Experiment 1, since uppercase text is known to be read more slowly than lowercase (Tinker, 1963). The mean words per minute for Experiment 2 are significantly different from one another $[F(4,96)=11.39$, $p<.0001]$. The control condition was read significantly more quickly than each of the experimental conditions (HSD $=26.64, p<.05$ ).

The data were expressed as differences from the control mean, and these scores were entered into a two-way within-subject ANOVA in the same way as in Experiment 1. A significant effect was found for the number of words in which letters were changed in size within each text $[F(1,24)=16.61, p<.0001]$. However, the main effect for the number of letters changed per word was not found to be significant $[F(1,24)=0.48$, n.s.], nor was there any significant interaction between the number of letters changed in size and the number of words that the size changes affected $[F(1,24)=0.70$, n.s. $]$. It appears that within both upper- and lowercase texts, changing the size of letters within words slows reading rate by disrupting the integrity of whole-word patterns, much more than by disrupting letter-feature information.

\section{Discussion}

When words are disrupted through changing the size of their constituent letters, reading rate is slowed significantly. The number of letters that are changed to cause the disruption has a relatively small effect. This experiment showed that this result is not specific to lowercase text, for the number of word patterns disrupted is also the significant factor in slowing reading rate when disrupted words are printed in uppercase. Whatever process is disrupted, it is not one making use of visual cues for word shape or word outline. 
We argued in the introduction that perceptual grouping must precede attention to words and that grouping may proceed through proximity or similarity. Since care was taken to ensure that the distance between words was always twice as great as the distance between letters, it is difficult to see how our manipulation could disrupt grouping by proximity. However, the similarity of the visual forms of the letters was changed on one dimension, scale. The letters of the alphabet are always different in form, which is how we discriminate between them, but generally they are similar in scale. Our hypothesis, therefore, is that letters of a similar scale are grouped together, and that attention is then directed to the group of letters that constitute the word, allowing further visual processing and lexical access. Letters of a differing scale disrupt this attentional grouping process.

\section{EXPERIMENT 3}

This experiment replicated Experiment 1, but with the letter sizes inverted; large letters were made small, and small letters large. On the basis of the hypothesis put forward to explain the results of Experiments 1 and 2, we expected the large letters, forming the major portion of the text, to be grouped together in the same way as the smaller ones were postulated to be grouped, and we expected that the major effect would be due to the number of words changed rather than the number of letters.

In Experiment 3, we employed the same materials and conditions as we did in Experiment 1. The only change was that the major portion of the text that was formerly in 9 points was now in 18, and the letters that were 18 points were now in 9. As in Experiment 1, either 125 or 250 words within each text were disrupted, this time through decreasing the size of their constituent letters, and either one or two letters were changed in size per word.

\section{Method}

The method was identical to that of Experiment 1, except for the changes described above. The subjects were 25 adult skilled readers. Their ages ranged from 24 to 55 years. They were all students of an undergraduate course in the Open University. They were paid fl each for their services.

One subject failed to complete the task, and 2 scored lower than criterion on the comprehension test. The appropriate three lines within the Latin square were reallocated to naive subjects, and they were subsequently included within the main experiment.

\section{Results}

Table 3 includes the mean comprehension scores for each condition. There were no significant differences between these means $[F(4,96)=.56$, n.s. $]$.

Mean reading rates are also presented in Table 3 in words per minute. They are significantly different $[F(4,96)$ $=7.16, p<.0001]$. An a posteriori comparison between the reading times per condition only demonstrated a significant difference between the control condition and the condition with 250 words disrupted and with two letters changed in size per word $(\mathrm{HSD}=37.03, p<.05)$. This reflects the larger standard deviations in this experiment.
Table 3

Mean Reading Rate in Words per Minute and Comprehension Scores for Each Condition in Experiment 3

\begin{tabular}{lcccc} 
& \multicolumn{2}{c}{ Words/Minute } & & \multicolumn{2}{c}{ Comprehension } \\
\cline { 2 - 3 } \cline { 5 - 6 } Condition & $M$ & $S D$ & $M$ & $S D$ \\
\hline Control & 235.56 & 90.33 & 4.08 & .64 \\
1L/125W & 222.16 & 95.66 & 4.20 & .76 \\
lL/250W & 214.14 & 86.03 & 4.28 & .68 \\
2L/125W & 200.27 & 74.55 & 4.00 & .82 \\
2L/250W & 170.26 & 61.57 & 4.12 & .78
\end{tabular}

Note-1L, one letter changed in size per word. $2 \mathrm{~L}$, two letters changed in size per word. $125 \mathrm{~W}, 125$ words changed per text. $250 \mathrm{~W}, 250$ words changed per text. $N=25$.

These data were transformed into difference scores as in Experiment 1 and entered into a two-way within-subject ANOVA. As in Experiment 1, a significant effect was found for the number of words in which letters were changed in size within each text $[F(1,24)=5.13, p<$ $.05]$. Unlike in Experiment 1, however, the main effect of the number of letters changed per word was also found to be significant $[F(1,24)=8.17, p<.01]$. No significant interaction was found between the number of letters changed in size and the number of words that the size changes affected $[F(1,24)=2.39$, n.s. $]$. It appears that decreasing the size of letters within words may slow reading rate by both disrupting the integrity of whole patterns and disrupting letter-level information.

\section{Discussion}

Experiment 3 demonstrates both configural and letterlevel effects. The main effect of the number of words changed cannot be explained merely as a concomitant of the significant main effect for the number of letters changed, because there was no significant interaction between these two variables. Thus, the configural effect observed in Experiments 1 and 2 was replicated in Experiment 3.

It is interesting to speculate why the significant effect of the number of letters changed, observed in Experiment 3 , was not found in the first two experiments, in which the changed letters were increased, rather than decreased, in size. One possibility is that small letters among large are more likely to suffer the effects of featural masking than large letters are among small.

To summarize, the effect of disruption in the word pattern is explicable in terms of the disruption of the smooth transition from perceptual grouping to selective attention and recognition, as well as the need to use a new strategy to integrate disparate items of relevant information. The effect of the number of letters changed is explained at the point of recognition. Changed letters may be more or less masked by adjacent letters, there being more masking with small letters among large.

Again, the critical assumption for this explanation is the hypothesis that groups are formed on the basis of the proportion of items at a particular size in the array. A larger proportion of items of a particular size dictates grouping of those items, as well as their subsequent at- 
tention and recognition. It also dictates the necessity of processing different-sized letters within that dominant group separately. It is hypothesized here that the effect of the number of word patterns disrupted, found in these first three experiments, is an index of perceptual grouping. It is further assumed that the group formed is based on the largest proportion of letters of similar size within a word.

\section{EXPERIMENT 4}

The important tenet of the grouping hypothesis is that letters that are similar in scale group together, making reading easier. So far, the question of when letters are deemed to be "similar" in size has not been discussed.

In computational models of vision, the representation of different size scales within the image is important as a cue to the existence of different objects. Such objects have a high probability of requiring separate attention during recognition (Marr, 1980; Watt, 1987). Concerning the issue of when size scales are similar and when they are dissimilar, psychophysical research has shown that visual input is processed by visual channels that are tuned to different sizes (Blakemore \& Sutton, 1969; Campbell \& Robson, 1968). At each point in the visual field, there are four channels that differ in size by a factor of two. The absolute size of these channels increases linearly from the fovea to the periphery, but their relative sizes remain roughly the same (e.g., Wilson, 1983; Wilson \& Berger, 1979).

From this psychophysical evidence, it would be expected that changing the scale of letters within words by a factor of two would require separate attention to, and processing of, those changed letters, and that reading may slow because of this need to process the changed letters separately from the whole. By this definition, letters that differ in scale from their neighbors by a factor of only 1.5 may be considered similar. Thus, a letter of 9 points is similar in scale to one of 14 points, and a 14-point letter is similar to one of 18 points, but 9 - and 18-point letters are dissimilar. It can therefore be argued that a 14point letter interposed between a 9-point and an 18-point letter may serve to permit grouping of the three. The present experiment was a test of this prediction.

In this experiment, Condition 1 was a control condition, as before, with all the texts in 18-point letters. In the first experimental condition, Condition 2, each adjacent letter was changed in size relative to its neighbor by a factor of 1.5. A 9-point letter was next to a 14-point letter, which was next to an 18-point, which was next to a 14-point. This pattern was repeated throughout the experimental texts, so that each letter was similar in scale to the next.

In the second experimental condition, Condition 3, the size of all the letters printed in 14 points in Condition 2 was changed to 9 points. Figure 2 provides samples of three types of text. There are fewer changes in size and less featural disruption in Condition 3 than in Condition 2,
Control

protozoan parasite called "Plasmodium". Although it has

Similar sizc

protozoan parasite called "Plasmodium". Al though it has

$$
\begin{aligned}
& \text { Different size } \\
& \text { parasite called "Plasmodilum". Aithough it has nOthing to } \mathrm{dl}_{0} \text { with }
\end{aligned}
$$

Figure 2. Samples from the materials used in Experiment 4.

but where changes in size do occur, the adjacent letters are markedly different in scale. A featural hypothesis would predict that Condition 3 , with the least amount of feature disruption, should be read most quickly, whereas the grouping hypothesis predicts the opposite. Condition 2 should be read most quickly because the letters are more similar in scale and therefore more readily grouped together.

\section{Method}

The method was identical to that of the previous experiments. Fifteen adult skilled readers participated in the experiment. Their ages ranged from 23 to 58 years. They were all students of an Open University foundation course and gave their time freely.

In this experiment, rather than selected words being changed as in the previous experiments, letters were changed continuously throughout the text regardless of word boundaries. In Condition 2, letters were changed in a regular fashion after the following manner: 9:14:18:14 (points). In this way, a 9-point letter was never adjacent to an 18-point letter. Condition 3 replicated the positions of both the 9- and the 18-point letters in Condition 2, but the 14point letters were changed to 9 points. Thus, Condition 3 had the same number of large 18-point letters as did Condition 2 , in the same positions, but a greater number of small, 9-point letters, and no 14-point letters.

\section{Results}

Table 4 includes the mean comprehension scores for each condition out of 5 . There are no significant differences between these scores $[F(2,28)=0.29$, n.s. $]$.

Mean reading rates are also presented in Table 4 . These times were entered into a one-way ANOVA. A significant main effect was found for conditions $[F(28,2)=13.10$, $p<.0001]$, and the control condition was read significantly more quickly than each of the experimental conditions (Tukey's HSD $=51.50, p<.05$ ]. As predicted, the similar-size condition was significantly faster than the different-size condition $[t(14)=2.267, p<.02]$.

\section{Discussion}

This experiment provides a direct comparison of the effects of feature disruption with the ease of perceptual grouping of letters. In Condition 2, each adjacent letter was changed in size scale, which also changed relative 
Table 4

Mean Reading Rate in Words per Minute and Comprehension Scores for Each Condition in Experiment 4

\begin{tabular}{lccccc}
\hline & \multicolumn{2}{c}{ Words/Minute } & & \multicolumn{2}{c}{ Comprehension } \\
\cline { 2 - 3 } \cline { 5 - 6 } Condition & $M$ & \multicolumn{1}{c}{$S D$} & & $M$ & $S D$ \\
\hline Control & 293.49 & 159.03 & 4.27 & .59 \\
Similar Size & 218.33 & 95.13 & 4.27 & .70 \\
Different Size & 187.24 & 85.25 & 4.13 & .64 \\
\hline
\end{tabular}

information about letter features. In Condition 3, fewer letters were changed in size, which led to less featural disruption in this condition. Using feature-based theories, we would therefore expect the latter condition to be read more quickly than the former. However, the variable of similarity in size scale was also manipulated. In Condition 2 , even though more letters were changed in size, each adjacent letter differed from its neighbor by a factor of only 1.5 , whereas in Condition 3 , fewer letters were changed, but they differed from their neighbor by a factor of two. Thus, in Condition 2, each letter was more similar in size to its neighbor than in Condition 3, and therefore, according to the hypothesis above, more amenable to grouping. Condition 2 was read significantly more quickly than Condition 3 .

\section{GENERAL DISCUSSION}

Much research in reading has been concerned exclusively with how readers recognize isolated words. Our experiments, on the other hand, look at how the processes leading to word recognition may be delayed by factors that disrupt aspects of early visual information processing. A framework for thinking about word recognition in reading within a broader context was outlined in the introduction. This drew heavily upon McConkie and Zola's (1987) account of segmentation and attention during reading. Such a model could only be viewed as a framework. It specifies what has to be computed, grouping some part of the visual field as the "page object," the "line object," or the "word object," attending to one of these and then recognizing features, letters, and finally the identity of the word. This framework requires a mechanism by which page, line, and word objects can be differentiated. One of the simplest ways to do this would be for the visual system to use the inherent characteristics of print to form the relevant grouping. For instance, words may be differentiated because they consist of elements that are proximal and similar in size. The results of our experiments, in which relative letter size within a word was changed, are interpreted in terms of disruption to this process.

Experiment 1 demonstrated a reduction in legibility that could not be explained as due to the disruption of subletter features. This finding, that legibility depended on the number of words mutilated, much more than on the number of letters changed, shifts the locus of explana- tion to the whole word. The effect was replicated in Experiment 3 with small letters inserted in a larger font, rather than large letters in a smaller font as in Experiment 1. In Experiment 3, there was evidence of a significant subletter feature effect. In Experiment 2, the effect was replicated with uppercase text. This rules out wholeword effects due to the disruption of supraletter features such as the word shape envelope of lowercase words. Taken together, Experiments 1 and 2 are difficult to explain in terms of disruption to any kind of feature detecting mechanism. Disruption of grouping effects is thus suggested by default of the alternatives.

This hypothesis was tested more directly in Experiment 4 . The two experimental conditions to be compared both consisted of a base text in a 9-point font with letters in an 18-point font interspersed in the same positions. Established principles of grouping would predict that flanking each 18-point letter with an intermediate-sized letter, here 14 points, would make it easier to group the 18-point letters with the 9-point. This is despite the fact that the visual system now had three rather than two letter sizes to cope with. This prediction was upheld.

A more detailed model of perceptual grouping processes is required to take this work further. In this connection, computational models such as MIRAGE (Watt \& Morgan, 1985) that make explicit the spatial arrangement of "bodies" within the visual field are most interesting. The MIRAGE algorithm uses Gaussian filters to distinguish between bodies in the visual field and the objects associated with those bodies. For example, a body might be a tree, its objects the branches or leaves. Similarity of size among the objects within a body is crucial to this process. In the event that further detail is required, Watt (1987) proposes a mechanism by which the elements that were objects can be turned into bodies containing their own objects, and so on. Empirical support for this computational model can be found in Watt (1988).

In terms of McConkie and Zola's (1987) account of segmentation and attention during reading, one might view lines as "objects" within a page "body." Similarly, words might be viewed as objects within a line body and letters as objects in a word body. This approach has the potential to give new insight into how word location and letter identity might be computed during reading. Because this is a computational model, it may be possible to make sophisticated quantitative predictions.

Clearly, this possibility is exciting and necessitates further research. Related to this is the need to establish clearly the relationship between computational grouping, subsequent attention to particular size scales, and recognition of the ensuing parsed structure. At a more general level, our approach calls for a much closer integration between research concerned with the cognitive mechanisms of reading and research concerned with basic visual processing. These two fields of endeavor have developed rapidly in the last 20 years, in virtual isolation from each other. 
It is clearly time for greater theoretical integration between these fields to occur.

\section{REFERENCES}

Blakemore, C., \& Sutton, X. X. (1969). Size adaptations: A new aftereffect. Science, 166, 245-274.

CAMPBell, F. W., \& RoBson, J. G. (1968). Contrast sensitivity, Fourier analysis and vision. Journal of Physiology, 197, 551.

CATTELL, J. M. (1886). The time taken up by cerebral operations. Mind, 11, 220-242.

Kahneman, D., \& Henik, A. (1975). Effects of visual grouping on immediate recall and selective attention. In S. Dornic (Ed.), Attention and performance VI (pp. 307-332). Hillsdale, NJ: Erlbaum.

KIмсHI, R. (1988). Selective attention to global and local levels in the comparison of hierarchical patterns. Perception \& Psychophysics, $\mathbf{4 3}$ 189-198.

KoFFKA, K. (1935). Principles of Gestalt psychology. New York: Harcourt Brace.

KOHLER, W. (1947). Gestalt psychology: An introduction to new concepts in modern psychology. New York: Liveright.

LARSEN, A., \& BUNDESEN, C. (1978). Size scaling in visual pattern recognition. Journal of Experimental Psychology: Human Perception \& Performance, 4, 1-19.

MARR, D. C. (1980). Visual information processing: The structure and creation of visual representations. Philosophical Transactions of the Royal Society of London, 290B, 199-218.

MCClelland, J. L., \& Rumelhart, D. M. (1981). An interactive activation model of context effects in letter perception. Psychological Review, 88, 375-407.

MCConkiE, G. W., \& Zola, D. (1987). Visual attention during eye fixations while reading. In M. Coltheart (Ed.), Attention and performance XII (pp. 385-401). Hillsdale, NJ: Erlbaum.

Monk, A. F., \& Hulme, C. (1983). Errors in proofreading: Evidence for the use of word shape in word recognition. Memory \& Cognition, 11, 16-23.

Navon, D. (1977). Forest before trees: The precedence of global features in visual perception. Cognitive Psychology, 9, 353-383.

PaAP, K., Newsome, S., \& Noel, R. (1984). Word shape's in poor shape for the race to the lexicon. Joumal of Experimental Psychology: Human Perception \& Performance, 10, 413-428.

Pomerantz, J. R. (1981). Perceptual organization in information pro- cessing. In M. Kubovy \& J. R. Pomerantz (Eds.), Perceptual organization (pp. 141-180). Hillsdale, NJ: Erlbaum.

Rayner, K., Pollatsek, A. (1989). The psychology of reading. Englewood Cliffs, NJ: Prentice-Hall.

Rudnicky, A. I., Kolers, P. A. (1984). Size and case of type as stimuli in reading. Journal of Experimental Psychology: Human Perception \& Performance, 10, 231-249.

Rumelhart, D. E., \& MCClelland, J. L. (1982). An interactive activation model of context effects in letter perception: II. Psychological Review, 89, 60-94.

SмITH, F. (1969). Familiarity of configuration vs discriminability of features in the visual identification of words. Psychonomic Science, $14,261,263$.

Smith, F., Lotr, D., \& Cronnell, B. (1969). The effect of type size and case alternation on word identification. American Journal of Psychology, 82, 248-253.

TAFT, M. (1991). Reading and the mental lexicon. London: Erlbaum. Tinker, M. A. (1963). Legibility of print. Ames, IA: Iowa State University Press.

Treisman, A. M. (1982). Perceptual grouping and attention in visual search for features and for objects. Journal of Experimental Psychology: Human Perception \& Performance, 8, 194-214

WATT, R. J. (1987). Scanning from coarse to fine spatial scales in the human vision system after the onset of a stimulus. Joumal of the $O p$ tical Society of America A, 4, 2006-2021.

WATT, R. J. (1988). Visual processing: Computational, psychophysical, and cognitive research. London: Erlbaum.

WATt, R. J., \& MORGAN, M. J. (1985). A theory of the primitive spatial code in human vision. Vision Research, 25, 1661-1674.

WERTHEIMER, M. (1923). Untersuchungen zur Lehre von der Gestalt, II. Psychologische Forschung, 4, 301-350. [Also translated as Laws of organization in perceptual forms. In W. D. Ellis (Ed.), A source book of Gestalt psychology (pp. 71-88). London: Routledge \& Kegan Paul, 1955]

WiLson, H. R. (1983). Psychophysical evidence for spatial channels. In O. J. Braddick \& A. C. Sleigh (Eds.), Physical and biological processing of images (pp. 88-99). Berlin: Springer-Verlag.

WiLson, H. R., \& BERGER, J. R. (1979). A four mechanism model for threshold spatial vision. Vision Research, 19, 19-32.

(Manuscript received March 20, 1992; revision accepted for publication July 28, 1992.) 